\title{
3D Multimodality Roadmapping in Neuroangiography
}

\author{
Daniel Ruijters ${ }^{a, c}$, Drazenko Babic ${ }^{a}$, Robert Homan ${ }^{a}$, Peter Mielekamp ${ }^{a}$, \\ Bart M. ter Haar Romeny ${ }^{b}$ and Paul Suetens ${ }^{c}$ \\ ${ }^{a}$ X-Ray Predevelopment, Philips Medical Systems, Veenpluis 6, 5680DA Best, the Netherlands \\ ${ }^{b}$ Biomedical Engineering, Image Analysis and Interpretation, Technische Universiteit \\ Eindhoven, Den Dolech 2, 5600MB Eindhoven, the Netherlands \\ ${ }^{c}$ Medical Image Computing, ESAT/Radiologie, Katholieke Universiteit Leuven, \\ Universitair Ziekenhuis Gasthuisberg, Herestraat 49, B-3000 Leuven, Belgium
}

\begin{abstract}
In this paper we describe a novel approach to using morphological datasets (such as CT or MR) in the minimally invasive image guidance of intra-arterial and intra-venous endovascular devices in neuroangiography interventions. Minimally invasive X-ray angiography procedures rely on the navigation of endovascular devices, such as guide wires and catheters, through human vessels, using C-arm fluoroscopy. While the bone structure may be visible, and the injection of iodine contrast medium allows to guide endovascular devices through the vasculature, the soft-tissue structures remain invisible in the fluoroscopic images. We intend to present a method for the combined visualization of morphological data, a 3D rotational angiography (3DRA) reconstruction and the live fluoroscopy data stream in a single image. The combination of the fluoroscopic image with the 3DRA vessel tree offers the advantage that endovascular devices can be located with respect to the vasculature, without additional contrast injection, while the position of the C-arm geometry can be altered freely. The additional visualization of the morphological data, adds contextual information to the position of endovascular devices. This article addresses the clinical applications, the real-time aspects of the registration algorithms and fast fused visualization of the proposed method.
\end{abstract}

Keywords: Image-Guided Therapy, Multimodality Display, Registration, Visualization

\section{INTRODUCTION}

To the present date, the fluoroscopic image with the live information about endovascular interventional devices, and morphological images (such as CT or MR) are visualized on separate displays. This means that the clinician has to perform a mental projection of the position of the endovascular device on the morphological data. It may be clear that a combined display of this information is of great advantage, since it reliefs the clinician of performing this task mentally, during the intervention. Furthermore, a fused image allows more precise navigation of the endovascular devices, since these devices are visualized together with pathologies and contextual information, present in the morphological data. In order to provide the maximum benefit of such an augmented image, the live fluoroscopy data and the morphological data have to be combined in real-time, with low latency and a sufficient frame rate ( 15 or 30 frames per second, depending on the acquisition mode). Since the visualization is targeted at the usage during an intervention, it should not only be fast, but also easy to interpret and the manipulation of the image should be interactive and easy to use.

In this article we describe the steps that are necessary to achieve such a combined visualization. Prior to fusing the 3DRA and morphological dataset, with the live fluoroscopy image stream, a pre-processing step is performed. In this pre-processing step the 3DRA and morphological dataset are registered, using an image-based registration algorithm, and the vessels are segmented from the 3DRA data. The pre-processing step is briefly touted in Sect. 3.1. During the visualization phase, an on-the-fly registration of the 2D fluoroscopy images and the $3 \mathrm{D}$ data has to be performed. This is achieved by using a machine-based registration, which only depends on the geometry incidence angles, the X-ray source to detector distance and the calibration data. The machinebased registration is described in Sect. 3.2. Section 3.3 discusses how a fast fused visualization of all three

E-mail: danny.ruijters@philips.com, Telephone: +31 402765742 
datasets can be implemented, using off-the-shelf graphics hardware. We discuss the clinical applications that can benefit from the presented work in Sect. 4. Section 5 describes the performance that we measured, using consumer hardware, and the conclusions are presented in Sect. 6. However, first we start with a review of the related work.

\section{RELATED WORK}

Two fundamentally different approaches can be distinguished, when registering the 2D fluoroscopy data to 3D volumetric data. In the first approach, called image-based registration, the registration process is driven by the image content. There are a number of image-based 2D-3D registration algorithms known in the literature for registering fluoroscopy images to either CT or MR images. ${ }^{1-7}$ These algorithms, however, take a considerable amount of time to compute. Further they need sufficient landmarks to be present in the 2D fluoroscopy image, which is not necessarily always the case.

The second approach is known as machine-based registration. With the introduction of motorized calibrated C-arm X-ray angiography, 3D reconstruction of the vasculature came within reach. Since such 3DRA datasets are obtained with the same apparatus as the 2D fluoroscopy data, it is possible to calculate a registration, based on the state of the geometry (viewing incidence angles, source-detector distance, etc.) and calibration data, provided that there was no patient motion between the acquisition of the 3DRA data and fluoroscopy data. ${ }^{8-10}$ This method also allows to obtain a registration, when there are insufficient landmarks present in the images (e.g. due to the absence of iodine contrast medium in the fluoroscopy images). A further advantage of machine-based registration is the fact that it can be computed in realtime. Machine based registration and image based 2D-3D registration have been compared by Baert et al. ${ }^{11}$ A method for determining the incidence based on tracking a fiducial was proposed by Jain et al. ${ }^{12}$ We, however, do not use any fiducials. We only use the information concerning the geometry state, as is provided by the C-arm system.

In earlier work, ${ }^{13}$ we already proposed the combined visualization of morphological data and vasculature, which was segmented in 3DRA reconstructions. Here, we intend to augment this data with the live fluoroscopy image stream, which enables the clinician to real-time correlate e.g. the guide wire or catheter position to the soft-tissue data.

\section{METHOD}

\subsection{Pre-processing}

Our approach relies on the acquisition of a 3DRA dataset, at the beginning of the intervention. The 3DRA dataset is registered to a morphological dataset, such as CT or MR, which has been obtained prior to the intervention (e.g. for diagnostic purposes). Using 3D image registration during interventional treatment poses a number of constrains on the registration algorithm. Especially, the calculation time of the algorithm has to be limited, since the result of the registration process is to be used during the intervention.

3DRA reconstructions may have a very high spatial resolution (a voxel can be as small as $0.1 \mathrm{~mm}$ ), but tend to be rather noisy in the dynamic range. Therefore the vessels, bones and sinuses are the only structures that are well visible, and can serve as landmarks. To reduce the sensibility to noise we use a limited number (16-32) of grey level bins for the 3DRA dataset.

Since we focus on cerebral applications, and there are only limited elastic transformations of the anatomical structures within the head, we can use a rigid registration (i.e. only a global translation and rotation). Rigid registration further has the property that it can be calculated relatively robust and fast. Typically, a registration algorithm consists of a multi-dimensional similarity measure, indicating the quality of a given spatial mapping, and an optimization algorithm, which searches the optimum (maximum or minimum, depending on the measure) of the similarity measure. The search space consists of the control variables of the similarity measure, which are in the case of rigid registration: translation in the $\mathrm{x}-, \mathrm{y}-$ and $\mathrm{z}$-direction, and rotation around the $\mathrm{x}-$, $\mathrm{y}-\mathrm{and}$ z-axis. We use Mutual Information as similarity measure, as described by Maes et al., ${ }^{14}$ because it performs very well on inter-modality registration and does not demand any a-priori knowledge of the datasets. In order to further limit the calculation time, we employ the Powell algorithm ${ }^{15}$ as optimizer, which is a so-called local 
optimizer. Local optimization algorithms are generally faster than global optimizers, but they do not guarantee that the overall optimum is found. To assure that the correct optimum is found, the image-based registration is preceded by an optional rough manual registration, which is to be performed by the clinician. Note that this pre-processing step has to be performed only once.

A further pre-processing step forms the creation of a triangulated mesh, representing the vessel tree. In order to obtain such a mesh, the vessels are segmented in the 3DRA volume, which is a fairly easy task since the iodine contrast medium absorbs more X-ray than any other substance present in the dataset. From the segmented data a mesh is extracted by applying the marching cubes algorithm. ${ }^{16}$

\subsection{D-3D Registration}

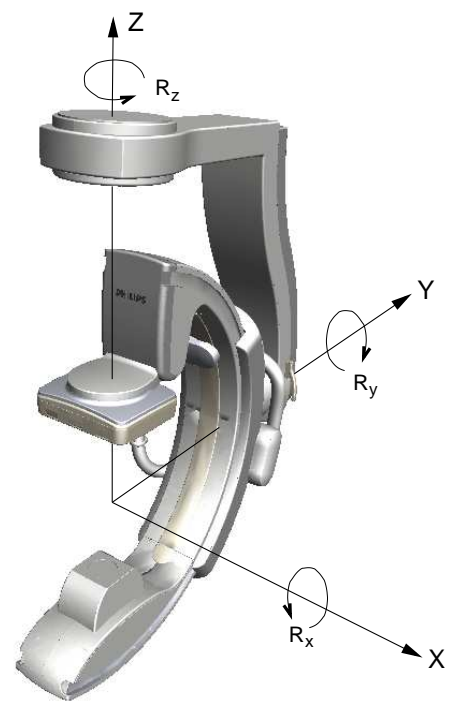

(a)

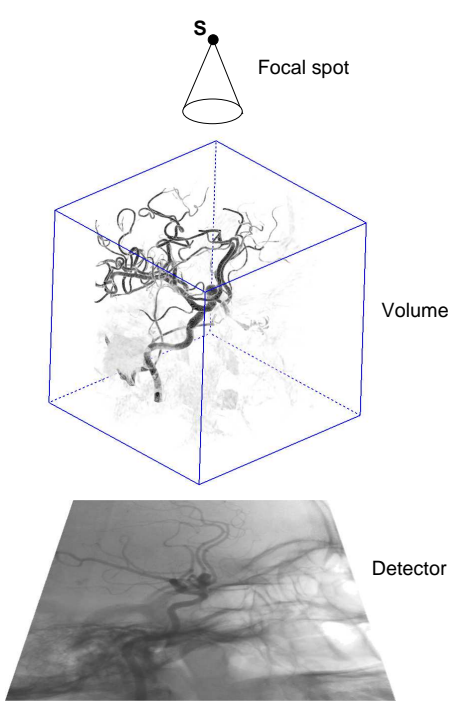

(b)

Figure 1. (a) the degrees of freedom of the C-arm geometry, (b) the virtual projection of a 3DRA dataset on a fluoroscopy image.

The machine-based registration involves determining the transformation of the coordinate space of the 3DRA data to the coordinate space of the fluoroscopy data. Let the origin of the coordinate system of the 3DRA data be positioned at the center of the dataset, and let the $\mathrm{x}$-axis correspond to the short side of the table, the $\mathrm{y}$-axis to the long side of the table, and the z-axis point from the floor to the ceiling. The X-ray C-arm system can rotate over three axis (see Fig. 1a). The rotation of the detector coordinate system, with respect to the table can be expressed as:

$$
\begin{gathered}
M=R_{x} \cdot R_{y} \cdot R_{z}= \\
\left(\begin{array}{ccc}
1 & 0 & 0 \\
0 & \cos \alpha & -\sin \alpha \\
0 & \sin \alpha & \cos \alpha
\end{array}\right) \cdot\left(\begin{array}{ccc}
\cos \beta & 0 & \sin \beta \\
0 & 1 & 0 \\
-\sin \beta & 0 & \cos \beta
\end{array}\right) \cdot\left(\begin{array}{ccc}
\cos \gamma & -\sin \gamma & 0 \\
\sin \gamma & \cos \gamma & 0 \\
0 & 0 & 1
\end{array}\right)
\end{gathered}
$$

Note that the order of the matrix multiplications is given by the mechanics of the C-arm system. The C-arm system's iso-center serves as origin for the rotation matrices. The rotation of 3DRA volume to the detector coordinate system corresponds to the inverse of matrix $M$, which is equal to its transposed, since rotation matrices are orthogonal. The matrix $M^{T}$ then has to be corrected for deviations from the ideally calculated orientation, based on the calibration data. ${ }^{17,18}$

After the rotation of the 3DRA dataset into the appropriate orientation, and a translation of the origin from the system's iso-center to center of the detector, there still remains the task of projecting it with the proper 
perspective (see Fig. 1b). The perspective depends on the X-ray source to detector distance (SID) and the detector dimensions. If the detector coordinate system uses the same metric as the coordinate system of the 3DRA dataset (e.g. millimeters), then the projection matrix will only depend on the SID. The projection matrix, which can be applied on homogenous coordinates $(x, y, z, w)$, can then be written as:

$$
P=\left(\begin{array}{cccc}
S I D & 0 & 0 & 0 \\
0 & S I D & 0 & 0 \\
0 & 0 & 0 & 0 \\
0 & 0 & -1 & S I D
\end{array}\right)
$$

\subsection{Visualization}

To achieve interactive frame rates, and a minimal latency we seek to harvest the vast processing power of modern off-the-shelf graphics hardware. This power can be accessed by using the DirectX or OpenGL API. In order to render an image, first the triangulated mesh, representing the vessels, is rendered in the frame buffer. Simultaneously the depths of the triangles are written in the z-buffer. A stencil buffer operation is defined to write a constant $S_{1}$ to the stencil buffer, for every pixel in the frame buffer that is filled by the mesh.

Consequently, a slab out of the morphological dataset is mixed into the scene using direct volume rendering (see Fig. 3b). The position, orientation and thickness of the slab can be altered by the clinician. The slab is rendered by evaluating the direct volume rendering equation for each pixel in the view port. The volume rendering equation can be approximated by the following summation: ${ }^{19,20}$

$$
i=\sum_{n=0}^{N}\left(\alpha_{n} c_{n} \cdot \prod_{n^{\prime}=0}^{n}\left(1-\alpha_{n^{\prime}}\right)\right)
$$

Whereby $i$ denotes the resulting color of a ray, $\alpha_{n}$ the opacity of the volume at a given sample $n$, and $c_{n}$ the color at the respective sample.

This summation can be broken down in $N$ iterations over the so-called over-operator, ${ }^{21}$ whereby the rays are traversed in a back to front order:

$$
C_{n+1}=\alpha_{n} \cdot c_{n}+\left(1-\alpha_{n}\right) \cdot C_{n}
$$

Here $C_{n}$ denotes the intermediate value for a given ray. After $N$ iterations, $C_{N}$ represents the final color of that particular ray. $N$ should be chosen such that every voxel is at least sampled once (we use two samples per voxel). Standard alpha blending, offered by DirectX or OpenGL, can be used to implement the over-operator. Equation (4) can be evaluated for all pixels in the frame buffer simultaneously, by using a set of $N$ textured slices, containing the slab data. In iteration $n$, the textured slice $n$ is then blended into the frame buffer, under the appropriate translation, rotation and perspective. Whereby the slices are processed in a back-to-front order, from the perspective of the viewer. After each iteration, every pixel in the frame buffer represent their respective $C_{n+1}$ value. $^{22}$

The triangulated mesh already is present in the frame buffer, when the textured slices are rendered. To mix the triangulated mesh and the direct volume rendering, we test the z-buffer at each iteration of the over operator. If the z-buffer test shows that, for a particular pixel, the position of the present sample of the ray is further away from the viewer than the triangle in the frame buffer, the frame buffer remains unchanged. The first sample that lies closer to the viewer, will take the present value of the frame buffer as input, which was written by rendering the triangulated mesh. In this way the color of the mesh is blended into the volume rendering equation at the appropriate place.

The registration matrix, which was calculated in the first pre-processing step, is applied to the position of the slices, which makes a resampling of the slab with the morphological data to the grid of the 3DRA data unnecessary, leading to a better image quality. ${ }^{23}$ Also while rendering the slab, a stencil buffer operation is defined to write constant $S_{2}$ to every pixel that receives a color value from the direct volume rendering process, with $\alpha>0$. The $S_{1}$ labels can be overwritten by this operation.

Finally the current fluoroscopy image is blended into the frame buffer. This is done in multiple passes. The action that is performed on a given pixel in a certain pass, is determined by the value in the stencil buffer. $S_{1}$ in 


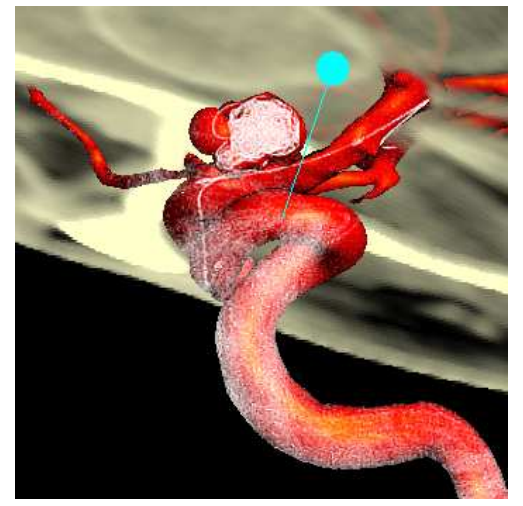

(a)

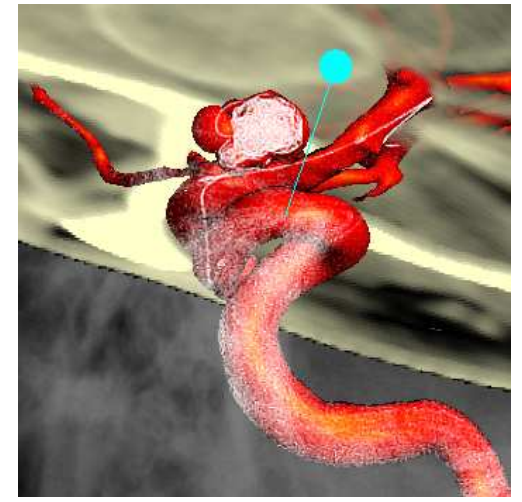

(b)

Figure 2. (a) In the first fluoroscopy overlay pass, the pixels that are labelled $S_{1}$ (vessel) in the stencil buffer, are treated. In this case, a sharpening filter was applied to the fluoroscopy data, before they were blended with the frame buffer content. (b) In the second pass, the pixels that were labelled as background in the stencil buffer, are processed. The fluoroscopy data is written without being sharpened, and the intensity is reduced.

the stencil buffer means that the vessel tree is depicted in that pixel, $S_{2}$ corresponds to the morphological data. If the stencil buffer is empty at a certain pixel position, then that particular pixel has not been filled with any information yet (background). Since the $S_{1}, S_{2}$ and empty regions are addressed individually, different blending and image processing operations can be performed to these regions (compare Figs. 2a and 2b). For instance, a spatial sharpening to enhance small details, and a temporal smoothing to reduce noise can be applied to the vessel region.

The fluoroscopy data that overlays the background, can contain some anatomical landmarks, which are relevant to the physician. The most important part of the fluoroscopy image, though, is to be found inside the vessel region, since the movement of the endovascular devices is supposed to be contained within this region. This hierarchy is reflected in the intensity and filtering of the fluoroscopy data stream. The fluoroscopic information that overlays the morphological slab could be suppressed, to reduce cluttering of information in this region.

\section{CLINICAL USE}

The availability of the live fluoroscopy image stream, combined with the vasculature, segmented from the 3DRA dataset and the registered morphological (CT or MR) dataset, during the intervention is of great clinical relevance. The combination of the fluoroscopy image with the 3DRA vessel tree provides the advantage that the guide wire and catheter position can be located with respect to the vessel tree, without additional contrast injection (see Fig. 3d), while the C-arm position and the X-ray source to detector distance can be altered freely. ${ }^{9}$ Even during e.g. rotations of the $\mathrm{C}$-arm, the machine-based 2D-3D registration will always be up to date. The additional visualization of the morphological data, allows to correlate the position of the guide wire and catheter to pathologies which are only visible in the morphological data. Especially the fact that this information is available in real-time, makes it very suitable for navigation.

The slab with the morphological data can be moved, its width can be changed and its orientation can be rotated freely, to visualize different parts of the anatomical dataset. In this way the optimal view of a certain pathology can be determined. The implementation of the rendering, running on the Graphics Processing Unit (GPU), offers interactive speed throughout.

The 3D multi-modality roadmapping method can be used in the following treatments: a) navigation to the optimal position for intra-arterial particle injection in endovascular embolization of intracranial neoplastic tissue, and arteriovenous malformation (AVM) treatment, prior to stereotactic radiation surgery, b) navigation to the optimal position for intracranial stenting in cases where aneurysms are pressing on surrounding eloquent and motoric brain tissue, c) navigation in the vessel portions to be embolized in e.g. hemorrhagic stroke, 


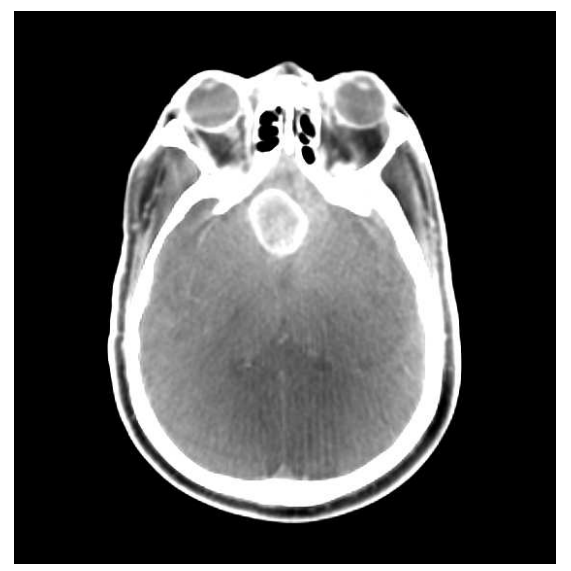

(a)

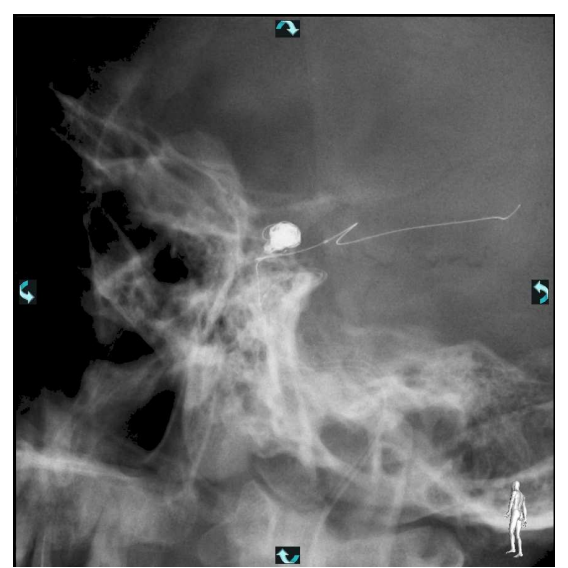

(c)

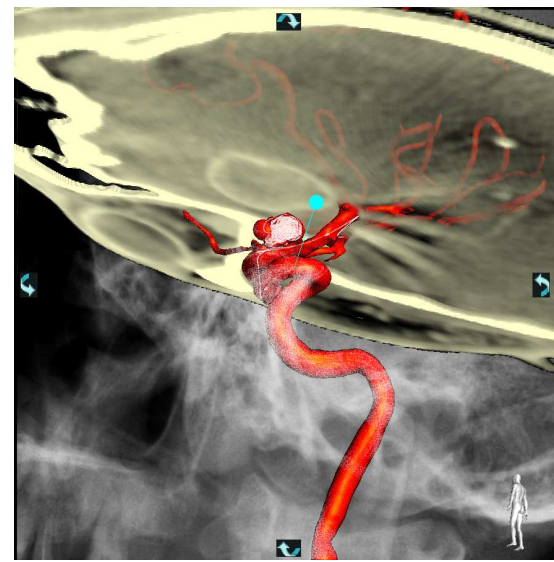

(e)

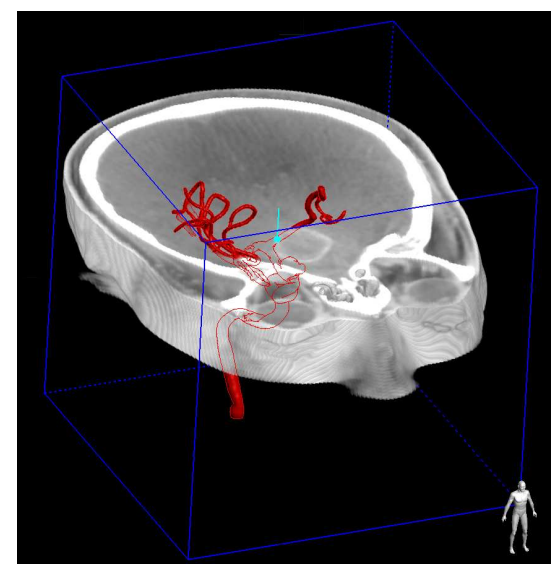

(b)

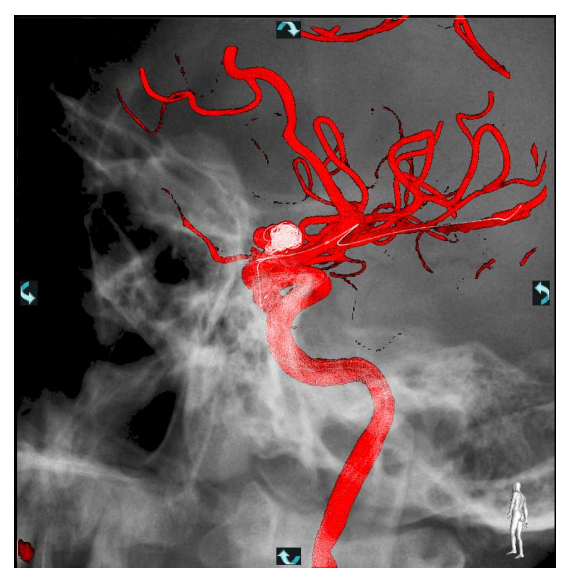

(d)

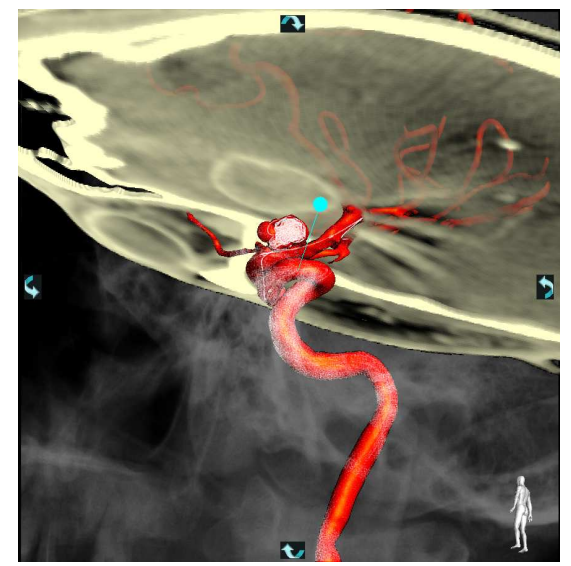

(f)

Figure 3. (a) A CT image, clearly showing a tumor, (b) The CT dataset, registered with the 3DRA dataset, (c) a single frame from the fluoroscopy image stream, (d) the fluoroscopy image mixed with the vessel tree from the 3DRA dataset, (e) the fluoroscopy image, the 3DRA vasculature and a slab from the CT data, (f) the fluoroscopy image outside the 3DRA vessel tree is darkened. 
d) navigation in the vessel segments where thrombolytic therapy should be applied in e.g. ischemic stroke or vascular vasospasms.

\section{RESULTS}

It takes 30 seconds to register the 3DRA dataset and the CT dataset in the pre-processing step, and $300 \mathrm{~ms}$ to calculate the mesh, which represents the vessels. Given a certain set of viewing incidence angles, it takes a mere $1.5 \mu s$ to calculate the matrix, which expresses the 2D-3D registration between the 3DRA dataset and the fluoroscopy image. The augmented visualization of a mesh extracted from a $256^{3}$ voxel 3DRA dataset, a Volume Rendered slab from a $256^{2} \cdot 198$ voxel CT dataset and the fluoroscopy image stream can be displayed at an average frame rate of 38 frames per second. All figures were measured on a Xeon 3.6 GHz machine with 2 GB of memory, and a nVidia QuadroFX 3400 graphics card with $256 \mathrm{MB}$ of memory, using the datasets that are depicted in Fig. 3. The accuracy of the calibrated machine-based 2D-3D registration was measured on 5 Philips Allura C-arm X-ray angiography systems. The registration was least accurate at the corners of the 3DRA reconstruction volume. The maximal deviation of the 2D fluoroscopy image and the projected 3DRA image was $0.4 \mathrm{~mm}$ at the corners of the reconstruction volume, and the average deviation at the corners was $0.2 \mathrm{~mm}$.

\section{CONCLUSIONS}

In this paper a method for the combined visualization of the cerebral blood vessels, segmented from 3DRA datasets, datasets containing the surrounding anatomy, such as CT or MR, and the live fluoroscopy data has been presented. The method is especially targeted at the use in minimally invasive vascular procedures, and distinguishes itself in the fact it adds contextual information to the fluoroscopy images and 3D vasculature.

The steps necessary to achieve this visualization have been described. First an image-based registration of the 3DRA dataset and the morphological dataset has to be performed. The machine-based registration between the fluoroscopy image and the 3DRA data only depends on the geometry incidence angles, the X-ray source to detector distance and the calibration data. It can be easily calculated in real-time. Also we described how the visualization can be implemented to employ the possibilities of modern off-the-shelf graphic cards, allowing real-time display of the registered data with the live fluoroscopy image stream. Further possible clinical applications have been identified, and it has been demonstrated how the presented method can be employed in those applications.

The strength of the described approach lies in its real-time nature, which is primarily achieved by the on-thefly 2D-3D registration, and the GPU-accelerated fused visualization. The interactive real-time aspect contributes to the 3D perception of the anatomy and pathologies during an intervention. We look forward to gain more clinical experience, which will help to quantify the benefits of the presented method in clinical performance measures (such as procedure duration), especially since this type of data fusing is new to the field of vascular interventions. A possible disadvantage of the present method is the fact that patient motion will render the 2D$3 \mathrm{D}$ registration to be invalid. Therefore future work could combine machine-based registration with image-based registration to correct for patient motion.

\section{ACKNOWLEDGMENTS}

We would like to thank the Rothschild foundation in Paris, France, and in particular Prof. Jacques Moret, for providing the depicted datasets.

\section{REFERENCES}

1. J. Weese, G. P. Penney, P. Desmedt, T. M. Buzug, D. L. G. Hill, and D. J. Hawkes, "Voxel-based 2-D/3-D registration of fluoroscopy images and CT scans for image-guided surgery," IEEE Trans. Information in Biomedicine 1(4), pp. 284-293, 1997.

2. A. Liu, E. Bullit, and S. M. Pizer, "3D/2D Registration Via Skeletal Near Projective Invariance in Tubular Objects," in MICCAI'98, pp. 952-963, 1998. 
3. G. P. Penney, P. G. Batchelor, D. L. G. Hill, D. J. Hawkes, and J. Weese, "Validation of a two- to threedimensional registration algorithm for aligning preoperative ct images and intraoperative fluoroscopy images," Med. Phys 28(6), pp. 1024-1031, 2001.

4. E. B. van de Kraats, G. P. Penney, D. Tomaževič, T. van Walsum, and W. J. Niessen, "Standardized Evaluation of 2D-3D Registration," in MICCAI'04, pp. 574-581, 2004.

5. G.-A. Turgeon, G. Lehmann, M. Drangova, D. Holdsworth, and T. Peters, "2D-3D registration of coronary angiograms for cardiac procedure planning," Med. Phys 32(12), pp. 3737-3749, 2005.

6. D. Tomaževič, B. Likar, and F. Pernuš, "3-D/2-D Registration by Integrating 2-D Information in 3-D," IEEE Trans. Medical Imaging 25(1), pp. 17-27, 2006.

7. G. Zheng, X. Zhang, and L.-P. Nolte, "A Class of Novel Point Similarity Measures based on MAP-MRF Framework for 2D-3D registration of X-Ray Fluoroscopy to CT Images," in ISBI'06, pp. 438-441, 2006.

8. J. B. A. Maintz and M. A. Viergever, "A Survey of Medical Image Registration," Medical Image Analysis 2(1), pp. 1-36, 1998.

9. M. Söderman, D. Babic, R. Homan, and T. Andersson, "3D roadmap in neuroangiography: technique and clinical interest," in Neuroradiology, (47), pp. 735-740, 2005.

10. S. Gorges, E. Kerrien, M.-O. Berger, Y. Trousset, J. Pescatore, R. Anxionnat, and L. Picard, "Model of a Vascular C-Arm for 3D Augmented Fluoroscopy in Interventional radiology," in MICCAI'05, pp. 214-222, 2005.

11. S. A. M. Baert, G. P. Penney, T. van Walsum, and W. J. Niessen, "Precalibration Versus 2D-3D Registration for 3D Guide Wire Display in Endovascular Interventions," in MICCAI'04, pp. 577-584, 2004.

12. A. K. Jain, T. Mustafa, Y. Zhou, G. S. Chirikjian, and G. Fichtinger, "FTRAC - A robust fluoroscope tracking fiducial," Med. Phys 32(10), pp. 3185-3198, 2005.

13. D. Ruijters, D. Babic, B. M. ter Haar Romeny, and P. Suetens, "Silhouette Fusion of Vascular and Anatomical Data," in ISBI'06, pp. 121-124, 2006.

14. F. Maes, A. Collignon, D. Vandermeulen, G. Marchal, and P. Suetens, "Multimodality Image Registration by Maximization of Mutual Information," IEEE Trans. Medical Imaging 16(2), pp. 187-198, 1997.

15. W. H. Press, S. A. Teukolsky, W. T. Vetterling, and B. P. Flannery, Numerical Recipes in C: The Art of Scientific Computing, Cambridge University Press, New York, NY, USA, 1992.

16. W. E. Lorensen and H. E. Cline, "Marching Cubes: A High Resolution 3-D Surface Construction Algorithm," ACM Computer Graphics 21(4), pp. 163-169, 1987.

17. M. Grass, R. Koppe, E. Klotz, R. Proksa, M. H. Kuhn, H. Aerts, J. op de Beek, and R. Kempkers, "Three-dimensional reconstruction of high contrast objects using c-arm image intensifier projection data," Computerized Medical Imaging and Graphics 23(6), pp. 311-313, 1999.

18. S. Gorges, E. Kerrien, M.-O. Berger, J. Pescatore, Y. Trousset, R. Anxionnat, S. Bracard, and L. Picard, "3D augmented fluoroscopy in interventional neuroradiology: Precision assessment and first evaluation on clinical cases," in MICCAI Workshop AMI-ARCS'06, 2006.

19. J. T. Kajiya, "The Rendering Equation," Computer Graphics, Proceedings of SIGGRAPH'86 20(4), pp. 143-150, 1986.

20. K. Engel, M. Kraus, and T. Ertl, "High-quality Pre-integrated Volume Rendering using HardwareAccelerated Pixel Shading," in Proceedings of the Eurographics workshop on Graphics hardware, pp. 9-16, ACM Press, 2001.

21. T. Porter and T. Duff, "Compositing Digital Images," ACM Computer Graphics 18(3), pp. 253-259, 1984.

22. D. Ruijters and A. Vilanova, "Optimizing GPU Volume Rendering," Journal of WSCG'06 14(1-3), pp. 9-16, 2006 .

23. K. J. Zuiderveld and M. A. Viergever, "Multi-modal Volume Visualization using Object-Oriented Methods," in ACM SIGGRAPH Symposium on Volume Visualization, pp. 59-66, 1994. 